\title{
Atypical Breast Myoepitheliosis
}

National Cancer Institute

\section{Source}

National Cancer Institute. Atypical Breast Myoepitheliosis. NCI Thesaurus. Code C40386.

A neoplastic process characterized by the proliferation of spindle to cuboidal

myoepithelial cells in and around small breast ducts exhibiting cytologic atypia and mitotic activity. 